\title{
Prevalence and Genetic Characterization of Carbapenem- and Polymyxin-Resistant Acinetobacter baumannii Isolated from a Tertiary Hospital in Terengganu, Malaysia
}

\author{
Soo-Sum Lean, ${ }^{1}$ Zarizal Suhaili, ${ }^{2}$ Salwani Ismail, ${ }^{3}$ Nor Iza A. Rahman, ${ }^{3}$ Norlela Othman, \\ Fatimah Haslina Abdullah, ${ }^{4}$ Zakaria Jusoh, ${ }^{4}$ Chew Chieng Yeo, ${ }^{2}$ and Kwai-Lin Thong ${ }^{1}$ \\ ${ }^{1}$ Institute of Biological Sciences, Faculty of Science, University of Malaya, 50603 Kuala Lumpur, Malaysia \\ ${ }^{2}$ Faculty of Agriculture, Biotechnology and Food Sciences, Universiti Sultan Zainal Abidin, 20400 Kuala Terengganu, Malaysia \\ ${ }^{3}$ Faculty of Medicine and Health Sciences, Universiti Sultan Zainal Abidin, 20400 Kuala Terengganu, Malaysia \\ ${ }^{4}$ Department of Pathology, Hospital Sultanah Nur Zahirah, 20400 Kuala Terengganu, Malaysia \\ Correspondence should be addressed to Kwai-Lin Thong; thongkl@um.edu.my
}

Received 5 December 2013; Accepted 9 February 2014; Published 19 March 2014

Academic Editors: B. Ayo, K. Leung, and C. Pazzani

Copyright (C) 2014 Soo-Sum Lean et al. This is an open access article distributed under the Creative Commons Attribution License, which permits unrestricted use, distribution, and reproduction in any medium, provided the original work is properly cited.

\begin{abstract}
Nosocomial infection caused by Acinetobacter baumannii is of great concern due to its increasing resistance to most antimicrobials. In this study, 54 nonrepeat isolates of A. baumannii from the main tertiary hospital in Terengganu, Malaysia, were analyzed for their antibiograms and genotypes. Out of the 54 isolates, 39 (72.2\%) were multidrug resistant (MDR) and resistant to carbapenems whereas 14 (25.9\%) were categorized as extensive drug resistant (XDR) with additional resistance to polymyxin B, the drug of "last resort." Pulsed-field gel electrophoresis analyses showed that the polymyxin-resistant isolates were genetically diverse while the carbapenem-resistant isolates were clonally related. The 14 XDR isolates were further investigated for mutations in genes known to mediate polymyxin resistance, namely, $p m r C A B$, and the lipopolysaccharide biosynthesis genes, $l p x A, l p x C$, $l p x D$, and $l p s B$. All 14 isolates had a $\mathrm{P} 102 \mathrm{H}$ mutation in $p m r A$ with no mutation detected in $p m r C$ and $p m r B$. No mutation was detected in $l p x A$ but each polymyxin-resistant isolate had 2-4 amino acid substitutions in $l p x D$ and 1-2 substitutions in $l p x C$. Eight resistant isolates also displayed a unique H181Y mutation in $l p s B$. The extent of polymyxin resistance is of concern and the novel mutations discovered here warrant further investigations.
\end{abstract}

\section{Introduction}

Acinetobacter baumannii is a Gram-negative bacterium increas-ing-ly found in the hospital environment due to its ability to survive for long periods on inanimate objects [1, 2]. Nosocomial A. baumannii isolates are mostly multidrug resistant (MDR) (i.e., resistant towards more than three classes of antibiotics) [3]. However, extensive drug resistant (XDR) isolates, which are resistant to all but one or two classes of antibiotics, and even pandrug resistant (PDR) isolates that are resistant to all classes of antibiotics, are developing at an alarming rate [4]. Since A. baumannii has the ability to colonize both viable and damaged tissues and is also resistant towards nearly all antimicrobials, it has become a cause of great concern. Carbapenems are among the very few antibiotics left that can be used for the treatment of $A$. baumannii infections. Nevertheless, the efficacy of carbapenems is increasingly compromised by the rapid emergence of carbapenem-hydrolysing $\beta$-lactamase enzymes. Several types of class D $\beta$-lactamases including OXA-23, OXA-24, OXA-58, and intrinsic OXA-51-like enzymes are known to be important contributors to carbapenem resistance [5].

The rapid development of carbapenem-resistant MDR A. baumannii has led to the use of polymyxins (in particular polymyxin B and colistin or polymyxin E) as the drug of "last resort" [6]. Polymyxins are cyclic, positively charged peptide antibiotics capable of posing antimicrobial activities to a broad variety of Gram-negative pathogens, including $A$. baumannii, due to their interaction with the lipid A moiety of lipopolysaccharide (LPS). This leads to the disorganization 
and disruption of the outer membrane integrity, causing cytoplasmic leakage [7]. Unfortunately, the intensive use of the polymyxins in recent years has led to the emergence of polymyxin heteroresistant and resistant A. baumannii, as high as 40.7\% reported in Spain [8] and 30.6\% in Korea [9].

The basis of polymyxin resistance in A. baumannii has only recently been investigated and several mechanisms have been proposed. Several genetic loci have been implicated in the resistance towards polymyxins in Acinetobacter, namely, the $p m r C A B$ operon $[10,11]$ and the $l p x A, l p x C, l p x D[12$, $13]$, and $l p s B$ genes, that are involved in LPS biosynthesis [14]. Resistance can arise through mutations in the twocomponent system PmrAB, in which the downstream target PmrC catalyzes the addition of phosphoethanolamine to the lipid A component of LPS [10,11, 15]. This modification reduces the net negative charge of the outer membrane thus reducing the affinity of polymyxins for the target. Park et al. (2011) [16] also showed that increased expression of $p m r A B$ could be essential for colistin resistance in A. baumannii. Mutations or insertions in the genes encoding the lipid A biosynthesis machinery, namely, the $l p x A, l p x C$, or $\operatorname{lp} x D$ genes, also mediate polymyxin resistance by abolishing the production of LPS, thereby eliminating the target of polymyxins $[12,13,17]$. Hood et al. (2013) [14] recently reported the identification of over 20 genes that are implicated in inducible colistin tolerance in A. baumannii, with majority of the genes involved in processes that protect the bacterium from osmotic stress. One of the genes identified, lps $B$, encodes a glycosyltransferase involved in LPS biosynthesis. Polymyxin resistance in A. baumannii thus developed through intrinsic mechanisms that respond and adapt to polymyxin treatment and not through acquisition of horizontally transferred resistance genes $[12,13]$.

To our knowledge, there has been no published data on polymyxin-resistant isolates of $A$. baumannii from Malaysia. In this study, we determined the resistance profiles of $A$. baumannii isolated from Hospital Sultanah Nur Zahirah (HSNZ), the main tertiary hospital located in the state of Terengganu in the east coast of Peninsular Malaysia. The genetic diversity of the A. baumannii isolates was determined by pulsed-field gel electrophoresis (PFGE). This study also aims to determine the presence and distribution of genes encoding the class $\mathrm{D}$ carbapenemase $b l a_{\mathrm{OXA}-51}, b l a_{\mathrm{OXA}-23}$, $b l a_{\mathrm{OXA}-24}$, and $b l a_{\mathrm{OXA}-58}$, as well as the class $\mathrm{B}$ carbapenemase (or metallo- $\beta$-lactamase) genes $b l a_{\mathrm{VIM}}$ and $b l a_{\mathrm{IMP}}$ in the clinical isolates of $A$. baumannii. Besides that, we also aim to investigate the type of mutations that occur within the $p m r C A B, l p x A, l p x C, l p x D$, and $l p s B$ genes that may confer resistance to polymyxin. Findings from this research project will help to determine the antimicrobial resistance, distribution of carbapenem and polymyxin resistance determinants, and the clonality of the A. baumannii isolates from HSNZ, thereby providing valuable information regarding this important nosocomial pathogen in this region.

\section{Materials and Methods}

2.1. Bacterial Isolates: Identification and Genotyping. Fiftyfour nonrepeat clinical isolates of A. baumannii were collected throughout 2011 from sporadic cases of infection in Hospital Sultanah Nur Zahirah (HSNZ). The isolates were isolated and initially identified by standard biochemical methods at the clinical microbiology laboratory. Single colonies of $A$. baumannii were then cultured into nutrient broth and incubated at $37^{\circ} \mathrm{C}$ for 24 hours. Confirmation of the 54 isolates was done by $16 \mathrm{~S}$ rDNA amplification and sequencing. Genomic DNA was extracted from the bacterial isolates using the Wizard Genomic DNA Purification Kit (Promega, Madison, WI, USA) and subjected to PCR using 16S rDNA universal primers [18] (Table 1). Strain typing of $A$. baumannii was carried out by pulsed-field gel electrophoresis (PFGE), as previously described [19]. Briefly, chromosomal DNA for PFGE analysis was prepared in agarose gel blocks, digested with restriction endonuclease ApaI (Promega, Madison, WI, USA). Restriction fragments were separated by using the CHEF Mapper (Bio-Rad, Hercules, CA, USA) with $0.5 \times$ TBE buffer for $26 \mathrm{~h}$ at $14^{\circ} \mathrm{C}$ with pulse times of 2-40 s. XbaI-digested Salmonella enterica ser. Braenderup H9812 was used as the molecular size standard. The PFGE profiles were analyzed using BioNumerics version 6.0 software (Applied Maths, Kortrijk, Belgium). The unweighted pair group method with averages (UPGMA), with a position tolerance for comparison of $1.5 \%$, was used to produce the dendrogram.

2.2. Antimicrobial Susceptibility: MIC Determination for Carbapenems and Polymyxin B. The Kirby-Bauer disc diffusion method was used to test the susceptibility of the A. baumannii isolates to the following 12 antibiotics (Oxoid Ltd., Basingstoke, UK): gentamicin (10 $\mu \mathrm{g})$, tobramycin $(10 \mu \mathrm{g})$, amikacin $(30 \mu \mathrm{g})$, ciprofloxacin $(5 \mu \mathrm{g})$, levofloxacin $(5 \mu \mathrm{g})$, piperacillin-tazobactam $(100 / 10 \mu \mathrm{g})$, cefotaxime $(30 \mu \mathrm{g})$, ceftazidime $(30 \mu \mathrm{g})$, cefepime $(30 \mu \mathrm{g})$, ampicillin-sulbactam $(10 / 10 \mu \mathrm{g})$, tetracycline $(30 \mu \mathrm{g})$, and doxycycline $(30 \mu \mathrm{g})$. The antibiotic susceptibility test was carried out on Mueller Hinton (Hi media) Agar swabbed with 0.5 McFarland standard suspension of $A$. baumannii and incubated at $37^{\circ} \mathrm{C}$ for $24 \mathrm{~h}$. Guidelines from the Clinical and Laboratory Standard Institute (CLSI) [20] were used to interpret the diameters of the inhibition zones produced.

Bacterial cultures of A. baumannii adjusted to $0.5 \mathrm{McFar}-$ land standard were used to determine the minimal inhibitory concentration (MIC) to imipenem and meropenem using M.I.C. Evaluator (M.I.C.E.) strips (Oxoid Ltd., Basingstoke, $\mathrm{UK}$ ). Inoculated media were incubated at $35 \pm 2^{\circ} \mathrm{C}$ for $16-$ $20 \mathrm{~h}$. The MIC values of the respective inhibition ellipses that interact with the strips were taken and recorded. Data were analyzed according to the guidelines given for M.I.C.E. strips and the MIC was determined.

MIC values for polymyxin B were determined by the agar dilution method [21]. Agar dilution plates were prepared by using Mueller-Hinton Agar added with concentrations of $2,4,8,16,32,64$, and $128 \mu \mathrm{g} / \mathrm{mL}$ polymyxin B sulfate (Sigma-Aldrich, St. Louis, MO, USA). A multipoint inoculator was used to deliver bacterial suspensions adjusted to $0.5 \mathrm{McF}$ arland standard onto the agar surface and the agar subsequently incubated at $35 \pm 2^{\circ} \mathrm{C}$ for $16-20 \mathrm{~h}$. The MIC value for polymyxin B was determined as the lowest 


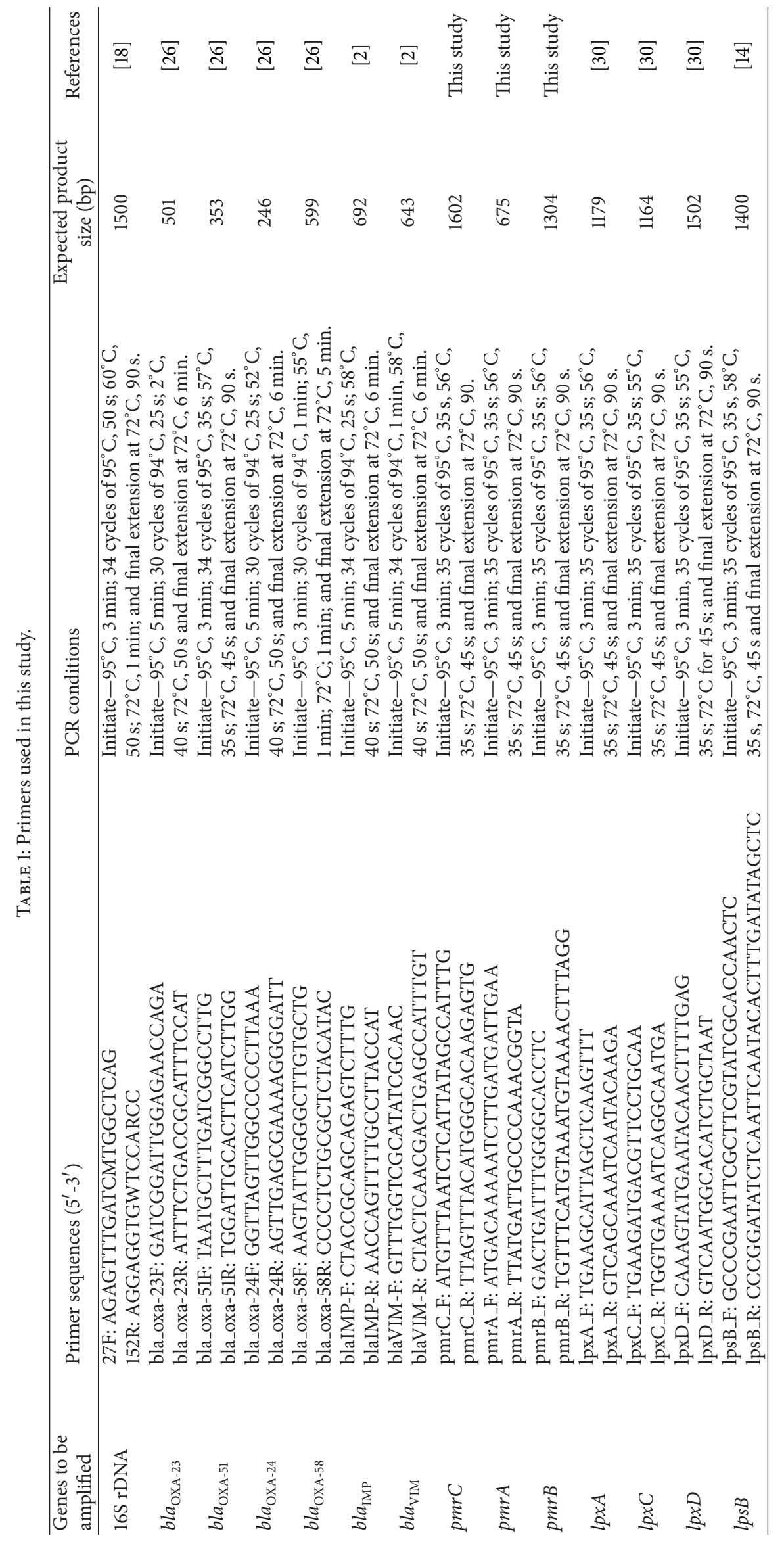


concentration of the antibiotic in which the bacteria were susceptible. The standard breakpoint for resistance according to the CLSI guideline [20] was $\geq 4 \mu \mathrm{g} / \mathrm{mL}$.

\subsection{Detection of Carbapenem- and Polymyxin-Resistant} Determinants, DNA Sequencing, and Sequence Analysis. The PCR primers and conditions used to detect the presence of several carbapenem resistance genes such as $b l a_{\text {OXA-23, }}$, $b l a_{\mathrm{OXA}-51}, b l a_{\mathrm{OXA}-24}, b l a_{\mathrm{OXA}-58}, b l a_{\mathrm{IMP}}$, and $b l a_{\mathrm{VIM}}$ are indicated in Table 1 . The validity of the amplicons was determined by DNA sequencing. Briefly, the amplified products were purified by using the Wizard SV Gel and PCR Clean-Up System (Promega, Madison, WI, USA) and then submitted to a commercial facility (First Base Sdn. Bhd., Malaysia) for sequencing.

Genetic determinants that have been implicated in conferring polymyxin resistance in $A$. baumannii, namely, the $p m r C A B, l p x A, l p x C, l p x D$, and $l p s B$ genes, were PCRamplified using specific primers and conditions as shown in Table 1. Purified PCR products were then sequenced at the commercial facility (First Base Sdn. Bhd., Malaysia).

DNA sequences obtained were edited using BioEdit v7.0.9 (downloadable from http://www.mbio.ncsu.edu/bioedit/ page 2.html) and analyzed with BLAST at the National Center of Biotechnology Information website (http://www.ncbi.nlm .nih.gov/BLAST). Sequence analysis was also done by using ExPASy translate tool at the Swiss Institute of Bioinformatics website (http://web.expasy.org/translate/) and ClustalW2 multiple sequence alignment program (http://www.ebi.ac .uk/Tools/msa/clustalw2/).

\section{Results}

3.1. Antibiotic Susceptibility Profiles of A. baumannii. DNA sequence analyses of the 16S rRNA amplicons showed $\geq 99 \%$ sequence identity with $A$. baumannii $16 \mathrm{~S}$ rRNA genes validating that all 54 isolates used in this study were A. baumannii.

High percentages of antibiotic resistance were observed for the following antibiotics: tetracycline (87\%), piperacillintazobactam (72.2\%), cefotaxime $(72.2 \%)$, ceftazidime (72.2\%), cefepime $(72.2 \%)$, levofloxacin $(70.4 \%)$, ampicillinsulbactam (68.5\%), gentamicin (66.7\%), ciprofloxacin $(66.7 \%)$, tobramycin $(64.8 \%)$, doxycycline $(61.1 \%)$, and amikacin $(57.4 \%)$. The isolates also showed high resistance for carbapenems with $77.8 \%$ of the isolates resistant to meropenem and $74.1 \%$ resistant to imipenem. The majority of the carbapenem-resistant isolates showed MIC values of $>32 \mu \mathrm{g} / \mathrm{mL}$. Of the 54 isolates, 39 (or $72.2 \%$ ) could be categorized as multidrug resistant (MDR) - that is, resistant to more than three classes of antibiotics. All MDR isolates were resistant to meropenem and imipenem. Polymyxin resistance was assessed by determining the MIC values for polymyxin B. Of the 54 isolates, 14 (or 25.9\%) were categorized as polymyxin resistant (MIC $\geq 4 \mu \mathrm{g} / \mathrm{mL}$ ). Four of these isolates had MIC values of $>128 \mu \mathrm{g} / \mathrm{mL}$ for polymyxin B. All 14 polymyxin-resistant isolates could also be categorized as extensive drug resistant (XDR) isolates based on the criteria proposed by Magiorakos et al. (2012) [22].
3.2. Detection of Class D and Class B Carbapenemase Genes. PCR was used to detect the presence of the class D carbapenemase genes $b l a_{\text {OXA-51 }}, b l a_{\text {OXA-23 }}, b l a_{\text {OXA-24 }}$, and $b l a_{\text {OXA-58 }}$. All $54 \mathrm{~A}$. baumannii isolates yielded $b l a_{\text {OXA-51 }}$ amplicons of the expected size of $353 \mathrm{bp}$. Sequence analyses of the amplified products confirmed their identity as $b l a_{\text {OXA-51 }}$ or $b l a_{\text {OXA-51-like }}$ gene. Forty-one (75.9\%) of the 54 isolates yielded

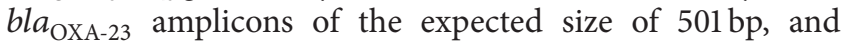
sequencing of the amplified products validated the identity as $b l a_{\text {OXA-23 }}$. None of the isolates harbor the $b l a_{\text {OXA-24 }}$ or the $b l a_{\text {OXA-58 }}$ genes. Similarly, no class B metallo- $\beta$-lactamase (MBL) genes, $b l a_{\mathrm{IMP}}$ or $b l a_{\mathrm{VIM}}$, were found in all the isolates tested.

3.3. Sequence Analyses of Polymyxin Resistance Determinants. All 14 polymyxin B resistant isolates were each subjected to PCR amplifications that target the $p m r C A B, \operatorname{lp} x A, \ln x C$, $l_{p} x D$, and $l p s B$ genes and the resulting amplicons were sequenced. The three isolates (AC6, AC11, and AC19) that were susceptible to polymyxin B were used as controls.

In this study, we found as many as 11 different point mutations within the $p m r B$ gene when compared to the reference isolates ATCC 17978 and ATCC 19606 but these mutations were similarly found in the polymyxin-sensitive isolates. Thus, when the $p m r B$ sequences of 14 polymyxinresistant isolates were compared with the 3 polymyxinsensitive isolates, no mutations within the $p m r B$ gene could be found. Similarly, when comparing the sequences of the response regulator gene $p m r A, 3$ different point mutations (R149K, T153I, and Q165P) were observed but these mutations were commonly found in both sensitive and resistant isolates. However, all 14 resistant isolates had a $\mathrm{P} 102 \mathrm{H}$ mutation within pmrA that was absent in the sensitive isolates (Table 2). When comparing the sequences for the $p m r C$ gene that encodes lipid A phosphoethanolamine transferase, up to 8 point mutations were found in comparison with the reference isolates. However no difference was found amongst the polymyxin-sensitive and polymyxin-resistant isolates.

The $l p x A, l_{p} C$, and $l p x D$ genes encode the first three enzymes in the lipid A biosynthesis pathway. No mutation was found in $l p x A$. In contrast, $l p x D$ showed up to 4 amino acid mutations in each resistant isolate while $l_{p x C}$ had 12 amino acid mutations (Table 2). The 14 resistant isolates had the K141R, S158R, or both mutations in $l p x C$ which encodes the enzyme involved in the second step of the lipid A biosynthesis pathway. The $l p x D$ gene showed the most number of mutations variation among the resistant isolates with a total of 13 different amino acid substitutions. Each resistant isolate had between 2 and 4 amino acid substitutions in $l p x D$ and most of the mutations occurred between amino acid residues 150 and 190 of LpxD. Further comparison of the $l_{p x D}$ sequence was carried out with known polymyxinsensitive isolates in the database and results of the multiple sequence alignment (data not shown) indicated that the amino acid substitutions found in our resistant isolates are unique.

The $\operatorname{lps} B$ gene encodes a glycosyltransferase involved in the biosynthesis of the LPS core and was recently implicated in A. baumannii colistin resistance [14]. Comparison of lpsB 
TABLE 2: Mutational points found in the polymyxin-resistant determinants among the 14 polymyxin-resistant A. baumannii isolates as compared to susceptible isolates.

\begin{tabular}{|c|c|c|c|c|}
\hline \multirow{2}{*}{ Isolate } & \multicolumn{4}{|c|}{ Mutations } \\
\hline & pmrA & $l p \times D$ & $\operatorname{lp} x C$ & $l p s B$ \\
\hline $\mathrm{AC} 12$ & $\mathrm{P} 102 \mathrm{H}$ & S102T, V141I, and R173G & K141R & H181Y \\
\hline AC30 & $\mathrm{P} 102 \mathrm{H}$ & S102T, V141I, and R173G & K141R & H181Y \\
\hline AC3 & $\mathrm{P} 102 \mathrm{H}$ & T104K, I178V & S158R & - \\
\hline $\mathrm{AC} 13$ & $\mathrm{P} 102 \mathrm{H}$ & T121A, N151D, and G169S & S158R & - \\
\hline $\mathrm{ACl} 6$ & $\mathrm{P} 102 \mathrm{H}$ & E50D, V141I & K141R & H181Y \\
\hline $\mathrm{AC} 17$ & $\mathrm{P} 102 \mathrm{H}$ & E50D, V141I & K141R & H181Y \\
\hline $\mathrm{AC} 18$ & $\mathrm{P} 102 \mathrm{H}$ & E50D, V141I & K141R & H181Y \\
\hline AC20 & $\mathrm{P} 102 \mathrm{H}$ & T15A, T121A, N151D, and G186S & K141R, S158R & H181Y \\
\hline $\mathrm{AC} 21$ & $\mathrm{P} 102 \mathrm{H}$ & T15A, T121A, N151D, and G186S & K141R, S158R & - \\
\hline $\mathrm{AC} 22$ & $\mathrm{P} 102 \mathrm{H}$ & E50D, S102T, and I178V & K141R, S158R & H181Y \\
\hline $\mathrm{AC} 27$ & $\mathrm{P} 102 \mathrm{H}$ & S102T, G169S, and R173G & S158R & H181Y \\
\hline AC38 & $\mathrm{P} 102 \mathrm{H}$ & S102T, G169S, I178V, and G186S, & K141R & - \\
\hline AC51 & $\mathrm{P} 102 \mathrm{H}$ & E50D, R173G & K141R, S158R & - \\
\hline AC61 & $\mathrm{P} 102 \mathrm{H}$ & $\mathrm{I} 178 \mathrm{~V}, \mathrm{~S} 181 \mathrm{~N}$ & K141R, S158R & - \\
\hline
\end{tabular}

${ }^{*}$ Note: no mutation was detected in $p m r C$, $p m r B$, and $l p x A$.

sequences among the 14 resistant isolates, the 3 sensitive isolates, and the 2 ATCC reference isolates initially showed that each resistant isolate contained as many as 4 amino acid substitutions in $l p s B$. However, when the $l p s B$ sequence from other polymyxin-sensitive isolates in the database was taken into account, the majority of these amino acid substitutions were found to be common among the other polymyxinsensitive isolates. The only unique mutation was H181Y and this substitution was found in 8 of the 14 resistant isolates (Table 2). The remaining 6 resistant isolates did not harbor any unique mutation within $l p s B$.

3.4. Genotyping of Acinetobacter baumannii Isolates by Pulsed-Field Gel Electrophoresis. PFGE analysis of the 54 ApaI-digested A. baumannii gave 15 reproducible profiles (pulsotypes) with a Dice coefficient, $F$, ranging from 0.90 to 1.00. Cluster analysis of the ApaI pulsotypes grouped the 54 A. baumannii isolates into 9 clusters with a cutoff point at $80 \%$ similarity (Figure 1). Carbapenem-resistant isolates were grouped into four main clusters, A-D, while carbapenemsusceptible isolates were grouped into two clusters, E and F.

Cluster A is the largest cluster with 21 carbapenemresistant isolates which were closely related ( $81.6 \%$ similarity), with 4 band differences at most $(F$ value $=0.90-1.00)$. Four of the isolates within the A cluster were also polymyxin resistant and XDR. In addition, all the carbapenem-resistant isolates in clusters A-D were all positive for $b l a_{\text {OXA-51-like }}$ and $b l a_{\text {OXA-23 }}$, whereas the susceptible isolates in clusters $\mathrm{E}$ and $\mathrm{F}$ were negative for $b l a_{\text {OXA-23 }}$. However, there was no clustering among pulsotypes of the polymyxin-resistant, XDR isolates. In fact, isolates with identical PFGE patterns would differ in their resistance to polymyxin B. For the three isolates in cluster $\mathrm{C}$ which shared identical PFGE patterns, two of these ( $\mathrm{AC17}$ and $\mathrm{AC18}$ ) were resistant to polymyxin $\mathrm{B}$ but the third isolate, $\mathrm{AC19}$, was polymyxin sensitive. Both $\mathrm{AC} 17$ and $\mathrm{AC} 18$ shared identical mutations within the polymyxin-resistance determinants (i.e., E50D and V141I in $l p x D, \mathrm{~K} 141 \mathrm{R}$ in $l p x C$, and $\mathrm{H} 181 \mathrm{Y}$ in lpsB). Likewise, AC29 and AC30 within cluster A shared an identical PFGE pattern but AC29 was sensitive to polymyxin B whereas AC30 was resistant.

\section{Discussions}

This study showed that the HSNZ isolates of A. baumannii were highly resistant to most antibiotics. Out of 54 isolates, $47(87 \%)$ were resistant to tetracycline, which was the highest resistance among the 12 antibiotics tested. The percentages of resistance for carbapenems were also high with $77.8 \%$ for meropenem and $74.1 \%$ for imipenem. Although the percentage of carbapenem resistance was lower when compared to the rates reported from another medical facility in Kuala Lumpur (96.5\% for imipenem and $98.2 \%$ for meropenem [19]), the finding that more than $50 \%$ of the isolates from HSNZ were carbapenem resistant is certainly a cause for concern as carbapenems have been the drugs of choice for Acinetobacter infections for over a decade [23, 24].

The overuse of carbapenems in hospitals to treat Acinetobacter infections has led to outbreaks of carbapenemresistant A. baumannii [25]. Outbreaks of isolates carrying genes encoding OXA-type carbapenemase have increasingly been reported worldwide in recent years [18]. There are eight subgroups of OXA carbapenemases, amongst which are the OXA-23-like, OXA-24-like, OXA-51-like, and OXA58-like groups [26]. The bla $a_{\text {OXA-51-like gene was found in }}$ all 54 A. baumannii isolates and this was to be expected as the gene is naturally occurring in A. baumannii where it may be overexpressed leading to carbapenem resistance [23]. Overexpression of $b l a_{\text {OXA-51 }}$ usually occurs due to the insertion of the ISAbal insertion sequence upstream of the gene which provides the strong promoter sequence [23]. Forty-one out of the 54 isolates of $A$. baumannii in this study 


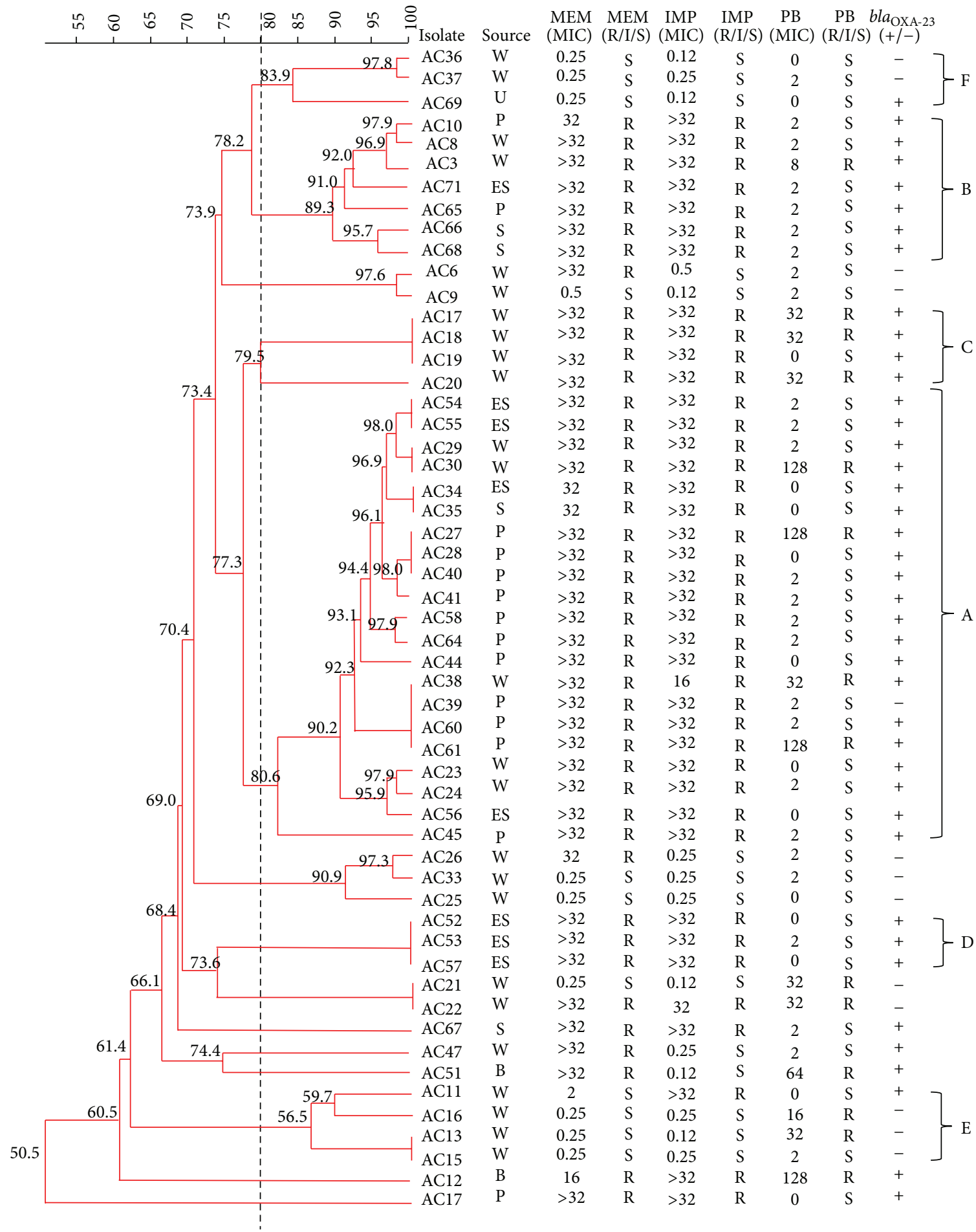

Figure 1: Dendrogram of the 54 A. baumannii isolates from Hospital Sultanah Nur Zahirah (HSNZ) using unweighted pair group arithmetic means methods (UPGMA) from ApaI PFGE profiles. The dotted vertical line indicates the cutoff point of $80 \%$ similarity. The sources of isolation for the A. baumannii isolates are as indicated (wound, W; pus, P; endotracheal secretion, ES; skin, S; urine, U; blood, B). The MIC values for imipenem (IMP), meropenem (MEM), and polymyxin B (PB) for the isolates are shown as are their susceptibility states (resistant, R; intermediate, I; susceptible, S) according to CLSI (2012) [20]. PCR detection results for bla $a_{\mathrm{OXA}-23}$ are also indicated ("+" for present; “-” for absent). The different clusters at $80 \%$ similarity are arbitrarily designated (A)-(E). 
were positive for the $b l a_{\text {OXA-23 }}$ gene. Of these 41 isolates, 37 were resistant to both meropenem and imipenem.

The second and third subgroups of carbapenem hydrolyzing $\beta$-lactamases were the OXA-24-like enzymes and OXA58-like enzymes [23] encoded by $b l a_{\text {OXA-24 }}$ and $b l a_{\text {OXA-58 }}$ genes, respectively. In this study, none of the 54 isolates were found to harbor the $b l a_{\text {OXA-24 }}$ and $b l a_{\text {OXA-58 }}$ genes, similar to recent findings from Thailand [27]. Likewise, none of the isolates carried the metallo- $\beta$-lactamase genes, $b l a_{\mathrm{IMP}}$ and $b l a_{\mathrm{VIM}}$, both of which have not been reported in $A$. baumannii in this region. However, reports from Korea $[28,29]$ showed that these two genes were implicated in carbapenem resistance.

The recent emergence of polymyxin-resistant and heteroresistant $A$. baumannii [6] has necessitated the need to understand the mechanism of the development of polymyxin resistance in this pathogen. Since this older drug has recently returned in use and has been noted as the drug of last resort for Acinetobacter infections [6], we investigated the antibiotic resistance of the HSNZ isolates towards polymyxin B. It was found that 14 of the 54 isolates (or 25.9\%) were resistant to polymyxin B, of which 4 had MIC values for polymyxin $\mathrm{B}>128 \mu \mathrm{g} / \mathrm{mL}$. The mechanism for polymyxin resistance in A. baumannii has only recently been investigated and the main mechanism appeared to be either covalent modification of the lipid A portion of LPS $[11,15]$ or disruption of LPS biosynthesis $[13,16]$. By modification and/or mutations in the amino acid sequences, negative charges on the outer membrane can be reduced, leading to reduction in the affinity of the positively charged polymyxin component, hence giving rise to polymyxin resistance $[10,11,15]$.

It is interesting that the 14 polymyxin-resistant isolates displayed an identical $\mathrm{P} 102 \mathrm{H}$ mutation within the $p m r A$ gene and this mutation was identical to that previously reported by Adams et al. (2009) [10] in a colistin-resistant derivative of $A$. baumannii AB0057. Overexpression of $p m r A B$ and mutations within these genes, especially $p m r B$, were reported to contribute to polymyxin resistance $[10,11,16]$. As expression levels of $p m r A B$ were not determined in this study and no mutation within $p m r B$ was found, we are not certain if the $\mathrm{P} 102 \mathrm{H}$ mutation in $p m r A$ is a contributing factor to the polymyxin resistance in the $14 \mathrm{XDR}$ isolates. However, analysis of the LPS biosynthesis genes identified novel mutations especially in the $l p x D, l p x C$, and $l p s B$ genes. All 14 XDR isolates had mutations in both $l p x D$ and $l p x C$ genes whereas 8 out of the 14 isolates showed an identical H181Y substitution in lpsB. It does appear that disruption in the LPS biosynthesis genes could be associated with polymyxin resistance in the A. baumannii isolates studied. The effects of these mutations on the LPS of these isolates need to be further investigated as they differ from the mutations previously reported [30]. Furthermore the crystal structure of the A. baumannii LpxD protein was recently elucidated [31] and one of the mutations (G186S) lies within the proposed active site of the enzyme. Nevertheless, it should be noted that in a recent study [14], screening of transposon mutant libraries led to the identification of more than 20 genes that may be involved in inducible colistin resistance in A. baumannii. Most of these genes converge on pathways involved in osmotolerance, cell envelope biosynthesis along with protein folding [14]. The role that these factors may play in the development of polymyxin resistance among the 14 XDR isolates would also need to be investigated.

PFGE results indicated that the polymyxin-resistant isolates were genetically diverse. This is in contrast to the carbapenem-resistant isolates which were clonally related. The carbapenem-resistant cluster A was the largest cluster inferring that this is the major clonal group of $A$. baumannii existing within the hospital in 2011. Four of the 21 isolates within the A cluster were polymyxin resistant and XDR with three of these isolates having MIC values for polymyxin B $>128 \mu \mathrm{g} / \mathrm{mL}$. Similar results were obtained whereby polymyxin-resistant isolates were found in clusters $\mathrm{E}, \mathrm{B}$, and $\mathrm{C}$ although the MIC values for these isolates were $\leq 32 \mu \mathrm{g} / \mathrm{mL}$. The clustering of carbapenem-resistant isolates has indeed been previously reported $[32,33]$. Carbapenem resistance is due mainly to the acquisition of carbapenemases such as OXA-23 which are encoded on plasmids and other mobile elements. Isolates that acquire such genes will then undergo clonal expansion $[34,35]$. In contrast, polymyxin resistance has been shown to develop through mutations in certain determinants in response to selection pressure [10-13, 17]. Thus these isolates were likely to develop randomly in the presence of polymyxins. Since polymyxin resistance is due to alterations or the complete absence of the outer membrane of LPS, it may incur a fitness cost to the bacterium. Therefore these isolates may not persist in the absence of polymyxin which could explain the random, nonclustering nature of the polymyxin-resistant isolates. This was indeed reported in a recent paper [36] where colistin-susceptible isolates outcompeted resistant isolates upon withdrawal of colistin in three out of four cases. However, in one case, an isolate with a $p m r B$ L271R mutation appeared to be fit enough to be transmitted in the absence of colistin and not be outcompeted by susceptible isolates [36]. Colistin-resistant $A$. baumannii has also been reported to have reduced virulence in mice [37] although a more recent report indicated contrasting results whereby colistin resistance may not necessarily intrinsically affect virulence [38].

This is the first report of polymyxin-resistant and XDR A. baumannii from Malaysia. The emergence of XDR and polymyxin-resistant $A$. baumannii isolates from the main tertiary hospital in Terengganu, Malaysia, is a cause of concern. Stricter enforcement of antibiotic usage and an antibiotic stewardship program are clearly needed in the hospital to prevent the spread of XDR A. baumannii.

\section{Conclusions}

Multidrug resistant A. baumannii was observed in 39 of 54 isolates $(72.2 \%)$ that were obtained from the main tertiary hospital in Terengganu, Malaysia, throughout 2011. High incidence of resistance to carbapenems (about 75\%) was noted. Of concern, 14 of the 54 isolates (25.9\%) were characterized as extensive drug resistant and were resistant to polymyxin B, considered the drug of "last resort." Pulsed-field gel electrophoresis indicated that the polymyxin-resistant isolates were genetically diverse. A $\mathrm{P} 102 \mathrm{H}$ mutation was 
found in the pmrA gene in all polymyxin-resistant isolates. In addition, novel mutations within the LPS biosynthesis genes $l p x C, l_{p x D}$, and $l_{p s} B$ were detected in the polymyxinresistant isolates. Whether these mutations contribute to the development of polymyxin resistance in A. baumannii requires further investigations.

\section{Conflict of Interests}

No competing financial interests exist.

\section{Acknowledgments}

The authors thank University of Malaya and Universiti Sultan Zainal Abidin for facilities and support and Hospital Sultanah Nur Zahirah (HSNZ) for providing the isolates used in this study. This project was supported financially by High Impact Research Grant UM.C/625/HIR/MOHE/02, 57-02-03-1015 and GA013-2013. Soo-Sum Lean is supported by University of Malaya Graduate Research Assistant Scholarship.

\section{References}

[1] E. Bergogne-Bérézin and K. J. Towner, “Acinetobacter spp. as nosocomial pathogens: microbiological, clinical, and epidemiological features," Clinical Microbiology Reviews, vol. 9, no. 2, pp. 148-165, 1996.

[2] T. S. Andriamanantena, E. Ratsima, H. C. Rakotonirina et al., "Dissemination of multidrug resistant Acinetobacter baumannii in various hospitals of Antananarivo Madagascar," Annals of Clinical Microbiology and Antimicrobials, vol. 9, no. 17, 2010.

[3] Y. K. Park, J. Y. Choi, D. Shin, and K. S. Ko, "Correlation between overexpression and amino acid substitution of the PmrAB locus and colistin resistance in Acinetobacter baumannii," International Journal of Antimicrobial Agents, vol. 37, no. 6, pp. 525-530, 2011.

[4] K. A. Davis, K. A. Moran, C. K. McAllister, and P. J. Gray, "Multidrug-resistant Acinetobacter extremity infections in soldiers," Emerging Infectious Diseases, vol. 11, no. 8, pp. 1218-1224, 2005.

[5] L. Poirel, T. Naas, and P. Nordmann, "Diversity, epidemiology, and genetics of class D $\beta$-lactamases," Antimicrobial Agents and Chemotherapy, vol. 54, no. 1, pp. 24-38, 2010.

[6] Y. Cai, D. Chai, R. Wang, B. Liang, and N. Bai, "Colistin resistance in Acinetobacter baumannii: clinical reports, mechanisms and antimicrobial strategies," Journal of Antimicrobial Chemotherapy, vol. 67, no. 7, pp. 1607-1615, 2012.

[7] R. E. Hancock, "Peptide antibiotics," The Lancet, vol. 349, no. 9049, pp. 418-422, 1997.

[8] L. A. Arroyo, I. Mateos, V. González, and J. Aznar, "In Vitro activities of tigecycline, minocycline, and colistin-tigecycline combination against multi- And pandrug-resistant clinical isolates of Acinetobacter baumannii group," Antimicrobial Agents and Chemotherapy, vol. 53, no. 3, pp. 1295-1296, 2009.

[9] K. S. Ko, J. Y. Suh, K. T. Kwon et al., "High rates of resistance to colistin and polymyxin $\mathrm{B}$ in subgroups of Acinetobacter baumannii isolates from Korea," Journal of Antimicrobial Chemotherapy, vol. 60, no. 5, pp. 1163-1167, 2007.

[10] M. D. Adams, G. C. Nickel, S. Bajaksouzian et al., "Resistance to colistin in Acinetobacter baumannii associated with mutations in the PmrAB two-component system," Antimicrobial Agents and Chemotherapy, vol. 53, no. 9, pp. 3628-3634, 2009.

[11] L. A. Arroyo, C. M. Herrera, L. Fernandez, J. V. Hankins, M. S. Trent, and R. E. W. Hancock, "The pmrCAB operon mediates polymyxin resistance in Acinetobacter baumannii ATCC 17978 and clinical isolates through phosphoethanolamine modification of lipid A," Antimicrobial Agents and Chemotherapy, vol. 55, no. 8, pp. 3743-3751, 2011.

[12] J. H. Moffatt, M. Harper, P. Harrison et al., "Colistin resistance in Acinetobacter baumannii is mediated by complete loss of lipopolysaccharide production," Antimicrobial Agents and Chemotherapy, vol. 54, no. 12, pp. 4971-4977, 2010.

[13] J. H. Moffatt, M. Harper, B. Adler, R. L. Nation, J. Li, and J. D. Boyce, "Insertion sequence ISAball is involved in colistin resistance and loss of lipopolysaccharide in Acinetobacter baumannii," Antimicrobial Agents and Chemotherapy, vol. 55, no. 6, pp. 3022-3024, 2011.

[14] M. I. Hood, K. W. Becker, C. M. Roux, P. M. Dunman, and E. P. Skaar, "Genetic determinants of intrinsic colistin tolerance in Acinetobacter baumannii," Infection and Immunity, vol. 81, no. 2, pp. 542-551, 2013.

[15] A. Beceiro, E. Llobet, J. Aranda et al., "Phosphoethanolamine modification of lipid A in colistin-resistant variants of Acinetobacter baumannii mediated by the PmrAB two-component regulatory system," Antimicrobial Agents and Chemotherapy, vol. 55, no. 7, pp. 3370-3379, 2011.

[16] Y. K. Park, K. R. Peck, H. S. Cheong, D. Chung, J. Song, and K. S. Ko, "Extreme drug resistance in Acinetobacter baumannii infections in intensive care units, South Korea," Emerging Infectious Diseases, vol. 15, no. 8, pp. 1325-1327, 2009.

[17] R. Henry, N. Vithanage, P. Harrison et al., "Colistinresistant, lipopolysaccharide-deficient Acinetobacter baumannii responds to lipopolysaccharide loss through increased expression of genes involved in the synthesis and transport of lipoproteins, phospholipids, and poly- $\beta-1,6-\mathrm{N}$ acetylglucosamine," Antimicrobial Agents and Chemotherapy, vol. 56, no. 1, pp. 59-69, 2012.

[18] R. Devereux and S. S. Wilkinson, "Amplification of ribosomal RNA sequences," in Molecular Microbial Ecology Manual, G. A. Kowalchuk, F. J. de Brujin, I. M. Head, A. D. L. Akkermans, and J. D. van Elsas, Eds., pp. 509-522, Kluwer Academic Publishers, Dordrecht, The Netherlands, 2nd edition, 2004.

[19] B. H. Kong, Y. A. Hanifah, M. Y. M. Yusof, and K. L. Thong, "Antimicrobial susceptibility profiling and genomic diversity of multidrug-resistant Acinetobacter baumannii isolates from a teaching hospital in Malaysia," Japanese Journal of Infectious Diseases, vol. 64, no. 4, pp. 337-340, 2011.

[20] Clinical and Laboratory Standards Institute, "Performance standards for antimicrobial susceptibility testing: twentysecond information supplement," CLSI Document M100-S22 [ISBN 1-56238-785-5]., Clinical and Laboratory Standards Institute, Wayne, Pa, USA, 2012.

[21] J. M. Andrews, "Determination of minimum inhibitory concentrations," Journal of Antimicrobial Chemotherapy, vol. 48, supplement 1, pp. 5-16, 2001.

[22] A.-P. Magiorakos, A. Srinivasan, R. B. Carey et al., "Multidrugresistant, extensively drug-resistant and pandrug-resistant bacteria: an international expert proposal for interim standard definitions for acquired resistance," Clinical Microbiology and Infection, vol. 18, no. 3, pp. 268-281, 2012. 
[23] P. Nordmann and L. Poirel, "Acinetobacter baumannii-basic and emerging mechanisms of resistance," European Infectious Disease, vol. 2, pp. 94-97, 2008.

[24] L. Poirel, T. Naas, and P. Nordmann, "Diversity, epidemiology, and genetics of class D $\beta$-lactamases," Antimicrobial Agents and Chemotherapy, vol. 54, no. 1, pp. 24-38, 2010.

[25] R. A. Bonomo and D. Szabo, "Mechanisms of multidrug resistance in Acinetobacter species and Pseudomonas aeruginosa," Clinical Infectious Diseases, vol. 43, supplement 2, pp. S49-S56, 2006.

[26] M. M. Feizabadi, B. Fathollahzadeh, M. Taherikalani et al., "Antimicrobial susceptibility patterns and distribution of BlaAXA genes among Acinetobacter spp. isolated from patients at Tehran hospitals," Japanese Journal of Infectious Diseases, vol. 61, no. 4, pp. 274-278, 2008.

[27] B. Thapa, C. Tribuddharat, S. Srifuengfung, and C. Dhiraputra, "High prevalence of bla $a_{\text {oxa-23 }}$ in oligoclonal carbapenemresistant Acinetobacter baumannii from siriraj hospital, Mahidol University, Bangkok, Thailand," Southeast Asian Journal of Tropical Medicine and Public Health, vol. 41, no. 3, pp. 625-635, 2010.

[28] S. H. Jeong, I. K. Bae, K. O. Park et al., "Outbreaks of imipenemresistant Acinetobacter baumannii producing carbapenemases in Korea," Journal of Microbiology, vol. 44, no. 4, pp. 423-431, 2006.

[29] J. Y. Sung, K. C. Kwon, J. W. Park et al., "Dissemination of IMP-1 and OXA Type $\beta$-Lactamase in Carbapenem-resistant Acinetobacter baumannii," Korean Journal of Laboratory Medicine, vol. 28, no. 1, pp. 16-23, 2008.

[30] J. H. Moffatt, M. Harper, P. Harrison et al., "Colistin resistance in Acinetobacter baumannii is mediated by complete loss of lipopolysaccharide production," Antimicrobial Agents and Chemotherapy, vol. 54, no. 12, pp. 4971-4977, 2010.

[31] J. Badger, B. Chie-Leon, C. Logan et al., "Structure determination of $L p x D$ from the lipopolysaccharide-synthesis pathway of Acinetobacter baumannii," Acta Crystallographica F, vol. 69, pp. 6-9, 2013.

[32] B. P. Chaulagain, S. J. Jang, G. Y. Ahn et al., "Molecular epidemiology of an outbreak of imipenem-resistant Acinetobacter baumannii carrying the ISAbal-bla $a_{\text {oxa-51 }}$-like Genes in a Korean hospital," Japanese Journal of Infectious Diseases, vol. 65, no. 2, pp. 162-166, 2012.

[33] Y. Kouyama, S. Harada, Y. Ishii et al., "Molecular characterization of carbapenem-non-susceptible Acinetobacter spp. in Japan: predominance of multidrug-resistant Acinetobacter baumannii clonal complex 92 and IMP-type metallo- $\beta$-lactamaseproducing non-baumannii Acinetobacter species," Journal of Infection and Chemotherapy, vol. 18, no. 4, pp. 522-528, 2012.

[34] S. D’Arezzo, L. Principe, A. Capone, N. Petrosillo, A. Petrucca, and P. Visca, "Changing carbapenemase gene pattern in an epidemic multidrug-resistant Acinetobacter baumannii lineage causing multiple outbreaks in central Italy," Journal of Antimicrobial Chemotherapy, vol. 66, no. 1, Article ID dkq407, pp. 5461, 2011.

[35] N. Karah, A. Sundsfjord, K. Towner, and Ø. Samuelsen, "Insights into the global molecular epidemiology of carbapenem non-susceptible clones of Acinetobacter baumannii," Drug Resistance Updates, vol. 15, no. 4, pp. 237-247, 2012.

[36] E. S. Snitkin, A. M. Zelazny, J. Gupta et al., "Genomic insights into the fate of colistin resistance and Acinetobacter baumannii during patient treatment," Genome Research, vol. 3, no. 7, pp. 1155-1162, 2013.
[37] R. López-Rojas, J. Domínguez-Herrera, M. J. McConnell et al., "Impaired virulence and in vivo fitness of colistin-resistant Acinetobacter baumannii," Journal of Infectious Diseases, vol. 203, no. 4, pp. 545-548, 2011.

[38] L. Lin, T. Brandon, P. Pantapalangkoor et al., "Inhibition of LpxC protects mice from resistant Acinetobacter baumnannii by modulating inflammation and enhancing phagocytosis," MBio, vol. 3, no. 5, Article ID e00312, 12 pages, 2012. 

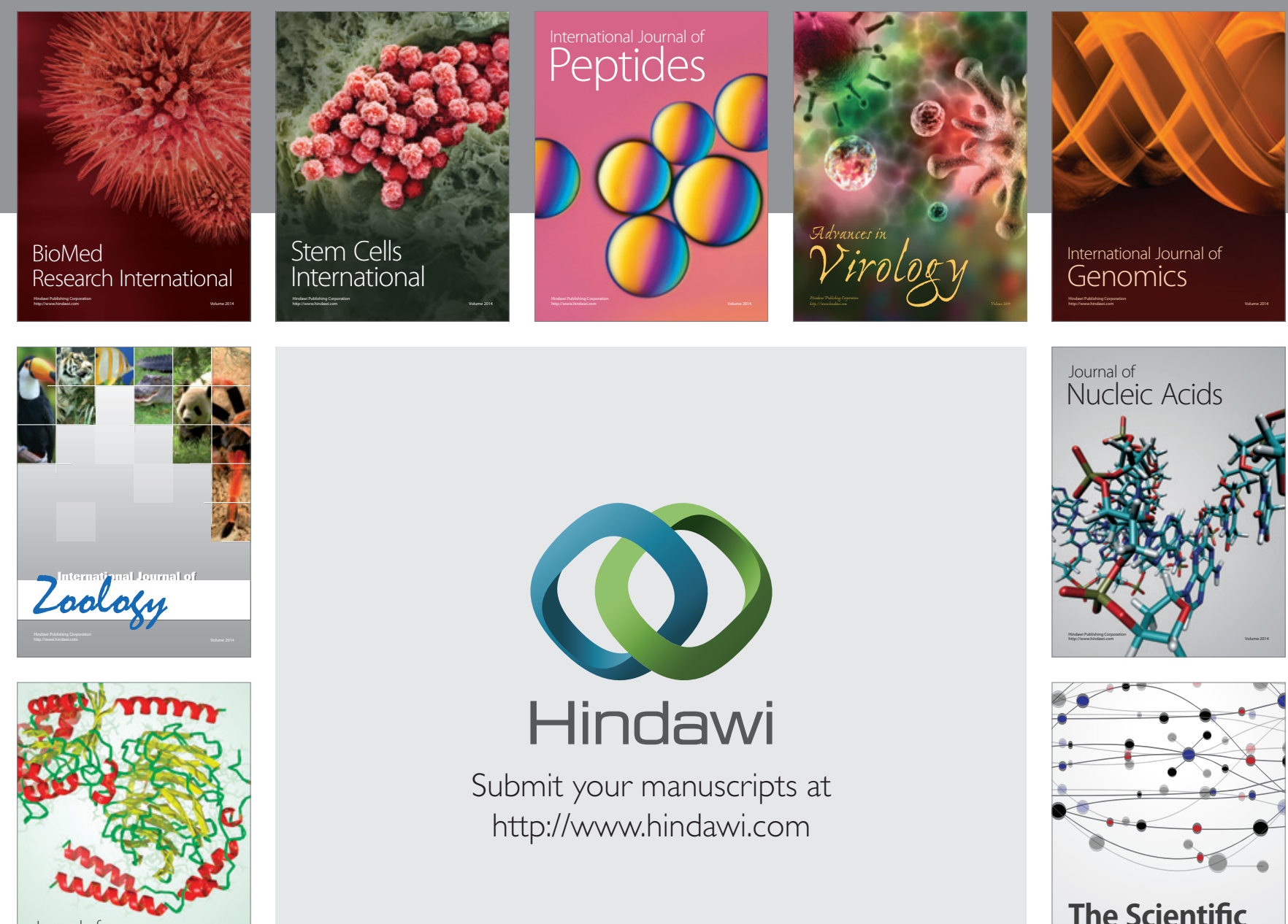

Submit your manuscripts at

http://www.hindawi.com

Journal of
Signal Transduction
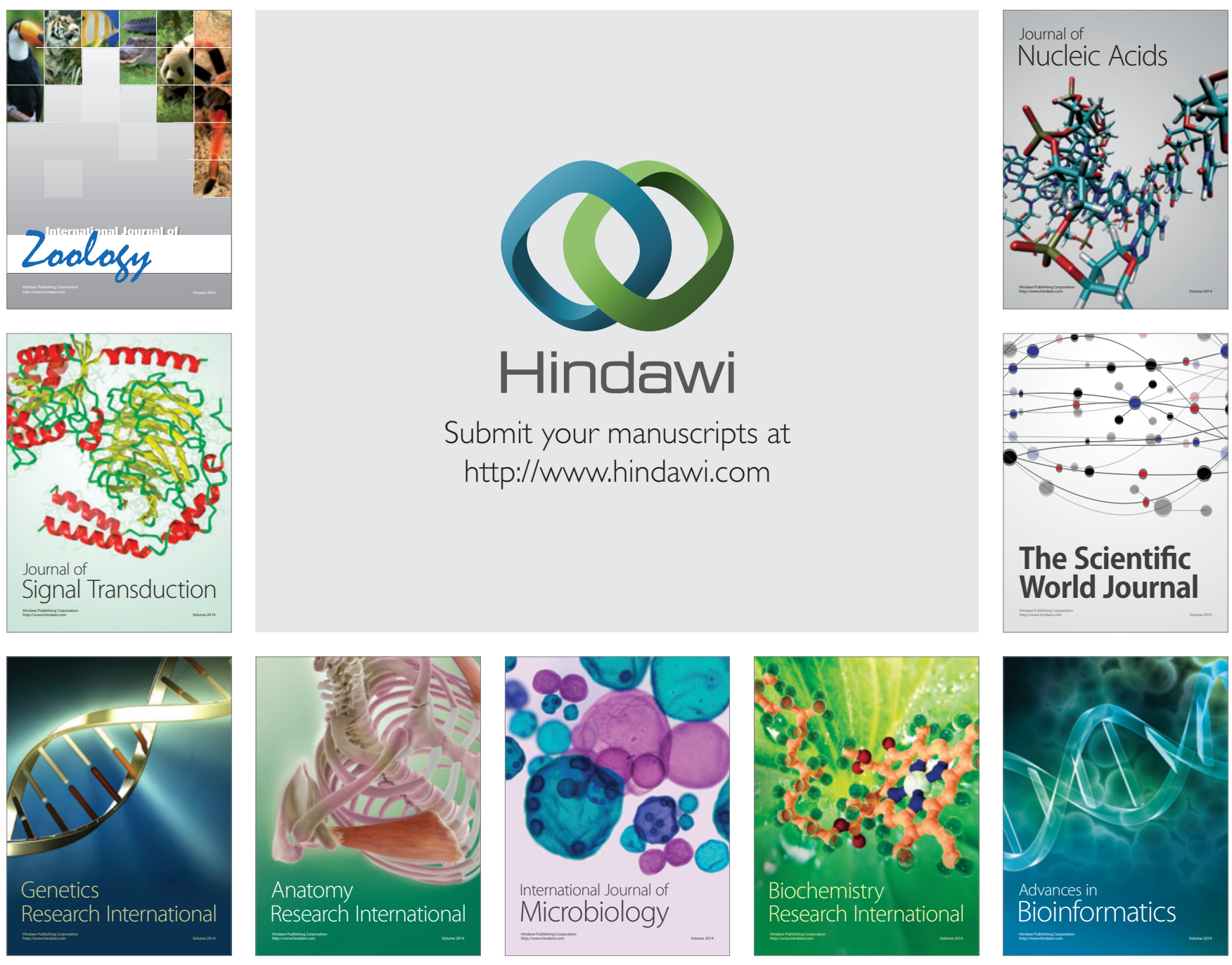

The Scientific World Journal
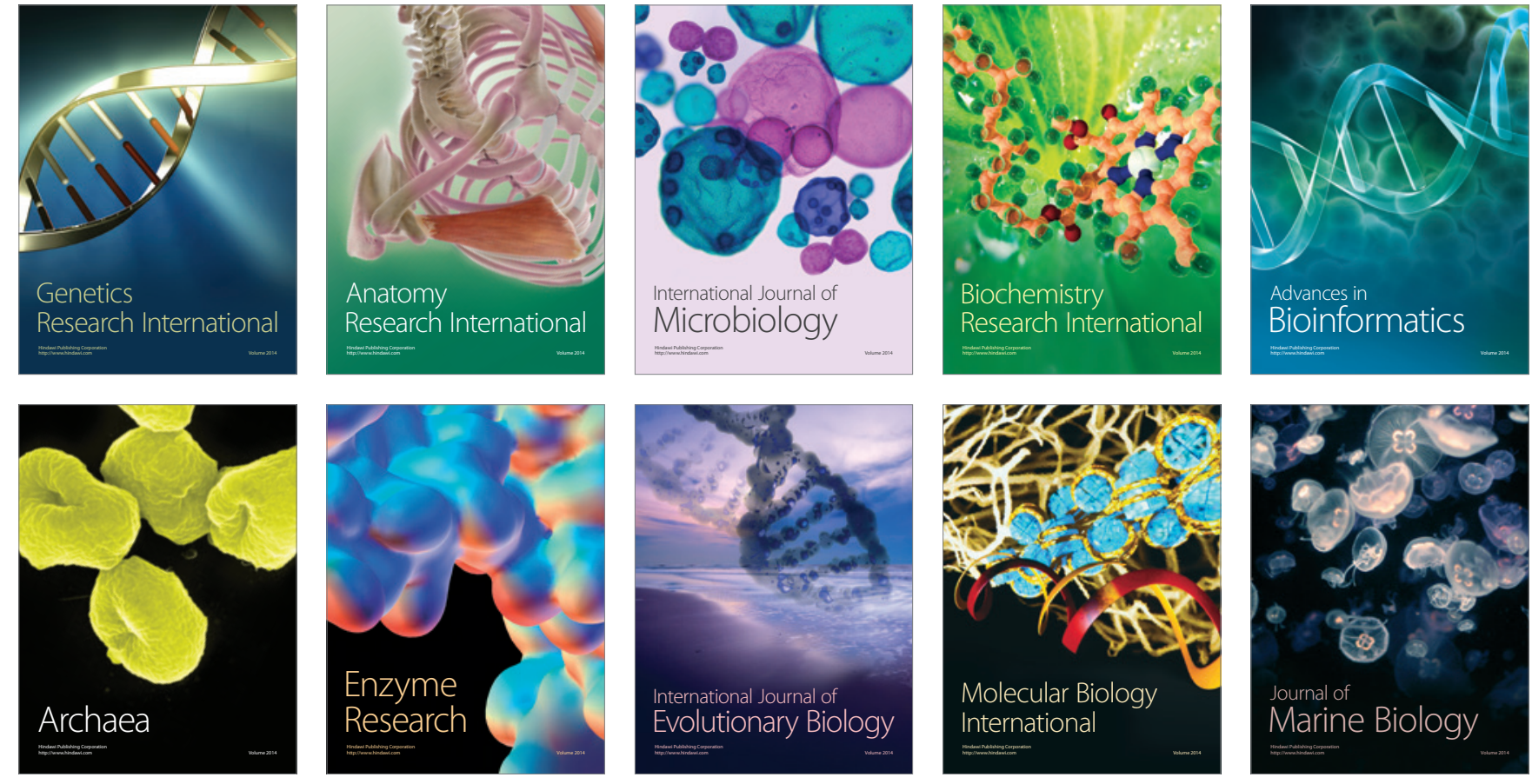